\title{
Initiation of Anti-dementia Medication in Older Adults Undergoing Pharmaceutical Management for Existing Chronic Conditions by the Assessment of Insurance Claims
}

Nobuhiro Handa ( $\square$ handa-nobuhiro@pmda.go.jp )

Pharmaceuticals and Medical Devices Agency https://orcid.org/0000-0003-2838-6462

\section{Seigo Mitsutake}

Tokyo Metropolitan Institute of Gerontology

Tatsuro Tatsuro Ishizaki

Tokyo Metropolitan Institute of Gerontology

Tetsuo Nakabayashi

Medicines and Healthcare Products Regulatory Agency

Masahiro Akishita

The University of Tokyo

Nanako Tamiya

Tsukuba Daigaku

Satoru Yoshie

The University of Tokyo

\section{Katsuya lijima}

The University of Tokyo

\section{Research article}

Keywords: Dementian, Insurance claims, Old adults, Alzheimer disease, Lewy body dementia

Posted Date: September 8th, 2020

DOI: https://doi.org/10.21203/rs.3.rs-65454/v1

License: (a) (1) This work is licensed under a Creative Commons Attribution 4.0 International License. Read Full License 


\section{Abstract}

BACKGROUND The influences of co-prescribed medications for chronic co-morbid conditions in very older adults on clinical dementia onset are uncertain.

OBJECTIVE To evaluate the influences of co-prescribed medications on clinical dementia onset, which was indicated by the initiation of a new prescription of anti-dementia medication (NPADM).

METHODS This retrospective cohort study consisted of 42024 adults aged $\geq 77$ years residing in a suburban city of Tokyo Metropolitan Area who did not take any anti-dementia medication on April 1, 2012.

The primary outcome was NPADM during followed-up period until March 31, 2015 (35 months). Subjects were categorized into four age groups: Group I (77-81 years), Group II (82-86 years), Group III (87-91 years), and Group IV ( $\geq 92$ years). Covariates were fourteen groups of medications prescribed between April 1, 2012 and June 31, 2012.

RESULTS In a follow-up period of 1345457 patient-months (mean=32.0 \pm 7.5 months), NPADM occurred in 2365 subjects. NPADM incidence at 12 months was $1.9 \pm 0.1 \%$ (Group I: $0.9 \pm 0.1 \%$, Group II: $2.1 \pm 0.1 \%$, Group III: $3.2 \pm 0.2 \%$ and Group IV: $3.6 \pm 0.3 \% ; P<0.0001)$. In addition to older age and female sex, the use of the following medications was associated with NPADM: statins (hazard ratio: 0.82, 95\% confidence interval: 0.73-0.92; $P=0.001$ ), antihypertensives $(0.80,0.71-0.85 ; P<0.0001)$, non-steroidal bronchodilators $(0.72,0.58-0.88 ; P=0.002)$, antidepressants $(1.79,1.47-2.18 ; P<0.0001)$, post-stroke medications $(1.45,1.16-1.82 ; P=0.002)$, insulin $(1.34,1.01$ 1.78; $P=0.046)$ and antineoplastics $(1.12,1.01-1.24 ; P=0.035)$

CONCLUSIONS These findings provide a benchmark for the management of dementia in the real-world setting, and contribute to evidence-based healthcare policymaking.

(Clinical Trial Registration: UMIN-CTR UMIN000039040)

\section{Background}

Dementia is a chronic neurodegenerative disorder that slowly progresses over years, and is characterized by cognitive decline, memory loss, and social functioning impairment. This condition often culminates in severe disability and dependency among older patients, thereby imposing an immense burden on their families and caregivers. Dementia is a serious global health problem, with approximately 50 million cases worldwide in 2015 and nearly 10 million new diagnoses annually. ${ }^{1}$ At this rate of increase, the number of people with dementia is expected to triple by $2050 .^{1}$

Current anti-dementia medications only work to alleviate symptoms, and there are no definitive treatment modalities to prevent or reverse the disease. ${ }^{2,3}$ Therefore, the prevailing goals of dementia management are to delay onset and slow its progression through the attenuation of risk factors. In 2019, the World Health Organization (WHO) issued guidelines to reduce the risks for cognitive decline and dementia, including recommendations for increased physical activity, increased social engagement, tobacco cessation, alcohol use reduction, nutritional management, weight management, cardiometabolic risk factor (hypertension, diabetes, and dyslipidaemia) management, and hearing loss management. ${ }^{1}$ However, the effects of these multimodal interventions are difficult to assess in real-world conditions due to the slow progression of cognitive decline and the need for multiple evaluations of patients over time. To understand the effects of such interventions, 
assessments must involve large numbers of participants, long-term observations, and regular medical examinations with standardized cognitive tests and/or imaging tests.

An integral component of Japan's universal healthcare system is the Long Life Medical Care System (LLMCS) (supplemental file 1), which provides insurance coverage to all Japanese residents aged 75 years or older and those aged 65 to 74 years with prespecified disabilities. ${ }^{4}$ LLMCS data encompass complete claims records of residents, including outpatient records, hospitalization records, prescription information, codes of medical supplies used during treatment, and medical procedure codes. As a result, LLMCS datasets can be applied to analyse the use of specific prescription medications in defined time periods. Many of the older adults covered by the LLMCS take numerous medications for existing conditions, including known dementia risk factors. ${ }^{5}$ Elucidating the associations between the pharmaceutical management of these risk factors and the progression to clinical dementia may shed light on the effectiveness of risk factor management on preventing disease onset.

In a complete enumeration of LLMCS data obtained from a suburban Japanese city, we examined if the use of prescription medications for existing chronic diseases is associated with the subsequent initiation of a new prescription of anti-dementia medication (NPADM). This study focused on medications which are indicated only for Alzheimer's disease and Lewy body dementia in Japanese regulatory approval.

\section{Methods}

This retrospective cohort study was conducted using insurance claims records of individuals residing in Kashiwa city-a typical satellite city with approximately 420,000 residents located in the suburbs of the Tokyo Metropolitan Area. Under Japan's universal healthcare system, citizens and foreign nationals residing in Japan for three months or longer are required to enrol in an insurance program. These include the LLMCS (for people aged $\geq 75$ years), national insurance programs provided by local governments (for unemployed, self-employed, and retired people aged $<75$ years), employees' insurance (for employed people aged $<75$ years and their dependents), and other various insurance programs (Table 1$).{ }^{4}$ 
Table 1

Patient sex and prescribed medications during the IBP in all subjects and according to age group

\begin{tabular}{|c|c|c|c|c|c|c|c|c|c|c|c|}
\hline & \multicolumn{2}{|c|}{ All subjects } & \multicolumn{9}{|c|}{ Age groups } \\
\hline & & & $77-81$ & years & $82-86$ & years & $87-91$ & years & $\geq 92 \mathrm{y}$ & ars & $P$ \\
\hline \multirow[t]{2}{*}{ No. of subjects } & 42024 & & 18232 & & 12346 & & 6942 & & 4504 & & \\
\hline & No. & $(\%)$ & No. & $(\%)$ & No. & $(\%)$ & No. & $(\%)$ & No. & $(\%)$ & \\
\hline Female & 23766 & 56.6 & 9405 & 51.6 & 6780 & 54.9 & 4252 & 61.3 & 3329 & 73.9 & $\begin{array}{l}< \\
0.0001\end{array}$ \\
\hline Insulin & 677 & 1.6 & 203 & 1.1 & 295 & 2.4 & 134 & 1.9 & 45 & 1 & $<.0001$ \\
\hline Oral antidiabetics & 3428 & 8.2 & 1072 & 5.9 & 1461 & 11.8 & 639 & 9.2 & 256 & 5.7 & $\begin{array}{l}< \\
0.0001\end{array}$ \\
\hline Statins & 7165 & 17 & 2194 & 12 & 3036 & 24.6 & 1407 & 20.3 & 528 & 11.7 & $<.0001$ \\
\hline Benzodiazepines & 3864 & 9.2 & 988 & 5.4 & 1530 & 12.4 & 884 & 12.7 & 462 & 10.3 & $\begin{array}{l}<.0001 \\
0.00\end{array}$ \\
\hline Antidepressants & 1064 & 2.5 & 250 & 1.4 & 429 & 3.5 & 264 & 3.8 & 121 & 2.7 & $<.0001$ \\
\hline Antiplatelets & 7007 & 16.7 & 1662 & 9.1 & 2647 & 21.4 & 1663 & 24 & 1035 & 23 & $\begin{array}{l}< \\
0.0001\end{array}$ \\
\hline Antianginals & 2021 & 4.8 & 382 & 2.1 & 683 & 5.5 & 514 & 7.4 & 442 & 9.8 & $\begin{array}{l}< \\
0.0001\end{array}$ \\
\hline Anticoagulants & 1507 & 3.6 & 382 & 2.1 & 600 & 4.9 & 355 & 5.1 & 170 & 3.8 & $\begin{array}{l}< \\
0.0001\end{array}$ \\
\hline Antihypertensives & 17237 & 41 & 4426 & 24.3 & 6619 & 53.6 & 3858 & 55.6 & 2334 & 51.8 & $\begin{array}{l}< \\
0.0001\end{array}$ \\
\hline Antineoplastics & 8011 & 19.1 & 2197 & 12.1 & 3257 & 26.4 & 1655 & 23.8 & 902 & 20 & $\begin{array}{l}< \\
0.0001\end{array}$ \\
\hline $\begin{array}{l}\text { Non-steroidal } \\
\text { bronchodilators }\end{array}$ & 2085 & 5 & 510 & 2.8 & 772 & 6.3 & 478 & 6.9 & 325 & 7.2 & $<.0001$ \\
\hline $\begin{array}{l}\text { Post-stroke } \\
\text { medications }\end{array}$ & 907 & 2.2 & 221 & 1.2 & 327 & 2.6 & 221 & 3.2 & 138 & 3.1 & $\begin{array}{l}< \\
0.0001\end{array}$ \\
\hline $\begin{array}{l}\text { NSAIDs, regular } \\
\text { use }\end{array}$ & 3205 & 7.6 & 679 & 3.7 & 1238 & 10 & 813 & 11.7 & 475 & 10.5 & $\begin{array}{l}< \\
0.0001\end{array}$ \\
\hline $\begin{array}{l}\text { Steroids, oral or } \\
\text { inhaled }\end{array}$ & 1685 & 4 & 461 & 2.5 & 673 & 5.5 & 385 & 5.5 & 166 & 3.7 & $<.0001$ \\
\hline
\end{tabular}

We obtained Kashiwa city's LLMCS insurance claims records from April 2012 to March 2015. These records were linked with the city's Basic Resident Register, which contains records of births, deaths, and movement of residence 
out of the city (which disqualifies an individual from the city's LLMCS). In the insurance claims records, age is categorized in 5-year intervals based on birth year (1921-1925, 1926-1930, 1931-1935, 1936-1940, and so forth). Although we sought to analyse residents aged 75 years or older (who qualify for LLMCS coverage), the 5year age categorization prevented the precise identification of these individuals. Therefore, we selected all residents aged 77 years or older (born in 1935 or earlier) on April 1, 2012 for inclusion in our study population. Because not all residents aged 72 to 76 years (born in 1936-1940) would be enrolled in the LLMCS, this age group was excluded from analysis.

The Medical Practitioners' Act of Japan mandates that physicians can only prescribe a medication for a maximum of three months at a time. For this analysis, we set an "initial background period" (IBP) of three months from April 1 to June 30, 2012 to identify each subject's baseline use of prescription medications for existing chronic diseases. From the 44302 eligible residents, those who had died $(n=365)$ or moved out of Kashiwa city $(n=129)$ during the IBP were excluded from analysis. In addition, 18 residents were excluded because they were residing in a nursing care facility on April 1, 2012, and were therefore covered by the long-term care insurance system (Supplemental file 1). We identified patients $(n=1766)$ who were already being treated with anti-dementia medication (cholinesterase inhibitors: donepezil, galantamine, and rivastigmine; N-methyl-d-aspartate receptor antagonist: memantine) for separate analysis. Accordingly, the main analysis was conducted on 42024 residents who were not treated with any anti-dementia medication during the IBP (Fig. 1).

The present research involving human data were performed in accordance with the Declration of Helsinki. The study used anonymized insurance claims records without opt-in informed consent, and was approved by the Kashiwa city government. The study was also approved by the institutional ethics committees of Tokyo Metropolitan Institute of Gerontology (Approval No28: October 16, 2015) where this study was performed.

\section{Identification of prescription medications}

Each subject's prescribed medications for existing chronic disease were identified using his/her insurance claims records. In order to be reimbursed under the LLMCS, healthcare providers submit claims to the Health Insurance Claims Review \& Reimbursement Services. This agency processes claims for pharmaceutical products approved for the Japanese market using specific billing codes. There were 200092 and 200597 billing codes for approved pharmaceutical products in 2012 and 2015, respectively. Medications for the dementia risk factors were categorized into 15 groups based on their pharmacological action; these included, among others, anti-dementia medications (215 codes), antidiabetics (482), antihypertensives (1762), statins (333), antidepressants (349), and benzodiazepines (157) (Supplimental file 2). Post-stroke medications that stimulate brain metabolism or increase cerebrovascular blood flow are also approved for use in patients with previous stroke, and were included in our analysis.

\section{Outcome measure and statistical analysis}

The outcome measure was NPADM. The first anti-dementia medication, donepezil, was approved for controlling the symptoms of Alzheimer's disease and Lewy body dementia in Japan by the Ministry of Health, Labour and Welfare in 1999. Since then, two more cholinesterase inhibitors (galantamine and rivastigmine) and one N-methyld-aspartate receptor antagonist (memantine) have been approved for the same indications. New prescriptions of any of these drugs were considered NPADM.

The observation period spanned a maximum of 35 months from April 2012 to March 2015. Subjects were censored if they died, moved out of Kashiwa city, or were admitted to a nursing care facility. As the insurance 
coverage of individuals shifted from the LLMCS to long-term care insurance after admission to a nursing care facility, their LLMCS insurance claims records would be incomplete after this shift. Therefore, these subjects were censored in the month of their admission to a nursing care facility. All subjects were stratified into four age groups (Group I: age 77-81 years, Group II: age 82-86 years, Group III: age 87-91 years, and Group IV: age $\geq 92$ years).

For the statistical analyses, continuous variables were calculated as mean values and standard deviations, and differences were evaluated using the unpaired $t$-test. Categorical variables were presented as frequency counts and proportions, and differences were evaluated using the likelihood ratio test. Cumulative incidence plots of NPADM for patient age, sex, and prescribed medications were generated using the Kaplan-Meier method with the log-rank test for comparisons. A Cox proportional hazards model was constructed for the multivariate analysis; patient age, sex, and 14 prescribed medications were included in the model as covariates. Results were expressed as hazard ratios and 95\% confidence intervals. All statistical analyses were performed using SPSS version 25 software (IBM Corp., Chicago, IL, USA). Two-tailed $P$-values below 0.05 were considered statistically significant.

\section{Results}

There were 1766 (4.0\%) subjects who were already taking anti-dementia medications during the IBP, and the proportion of these subjects increased with age (Group I: $1.1 \%$, Group II: $4.1 \%$, Group III: $8.0 \%$, and Group IV: $9.0 \%$ ). More than $56 \%$ of the 42024 main analysis subjects were taking prescription medications from one target medication group during the IBP, $21.9 \%$ were taking medications from three groups, and $4.1 \%$ were taking medications from five groups. The total follow-up period was 1345457 patient-months (mean \pm standard deviation: $32.0 \pm 7.5$ months, median: 35 months), with 34212 (81.4\%) subjects completing the full follow-up period of 35 months. NPADM occurred in 2365 (5.6\%) subjects. A total of 5447 (13.2\%) subjects were censored due to death $(n=3899)$, moving out of Kashiwa city $(n=669)$, or admission to a nursing facility $(n=879)$. The incidence of NPADM at 12 months was $1.9 \pm 0.1 \%$ (Group I: $0.9 \pm 0.1 \%$, Group II: $2.1 \pm 0.1 \%$, Group III: $3.2 \pm 0.2 \%$, and Group IV: $3.6 \pm 0.3 \%$; $P<0.0001$ ) (Fig. 2a). Women had a higher incidence of NPADM (Fig. 2b). The proportions of women and prescribed medications during the IBP according to age group are presented in Table 3. Approximately $56.6 \%$ of the subjects were women. During the IBP, $17.0 \%$ of subjects were taking statins, $41.0 \%$ were taking antihypertensives, $9.1 \%$ were taking antidiabetics (either insulin, oral hypoglycaemic agents or both), $2.5 \%$ were taking antidepressants, and $2.2 \%$ were taking post-stroke medications (Table 3 ).

The Kaplan-Meier analysis results (Figs. 2 to 5 ) showed that subjects who were taking the following medications during the IBP had significantly higher NPADM incidence: post-stroke medications $(P<0.0001)$, antidepressants $(P$ $<0.0001)$, antineoplastics $(P<0.0001)$, antiplatelets $(P=0.005)$, insulin $(P=0.019)$, and antianginals $(P=0.025)$. The univariate analysis did not detect any association between the use of antihypertensives or statins during the IBP and NPADM. The results of the Cox proportional hazards analysis (Fig. 6) showed that NPADM incidence was significantly associated with age, sex, and the following prescribed medications during the IBP: statins (hazard ratio: $0.82,95 \%$ confidence interval: $0.73-0.92 ; P=0.001)$, antihypertensives $(0.80,0.73-0.88 ; P<0.0001)$, nonsteroidal bronchodilators $(0.72,0.58-0.88 ; P=0.002)$, antidepressants $(1.79,1.47-2.18 ; P<0.0001)$, post-stroke medications $(1.45,1.16-1.82 ; P=0.002)$, insulin $(1.34,1.01-1.78 ; P=0.046)$, and antineoplastics $(1.12,1.01-1.24$; $P=0.035)$.

\section{Discussion}


In this retrospective complete enumeration study of older adults residing in a Japanese city, we examined the associations between the use of prescription medications for existing chronic conditions (indicating the pharmaceutical management of dementia risk factors) and NPADM (indicating the progression to clinical dementia). The NPADM incidence was $1.9 \%$ at 12 months after study initiation, and this incidence in subjects aged 87 years or older was thrice that of younger subjects aged 77-81 years. NPADM incidence was significantly higher in women and subjects who were treated with insulin, antidepressants, antineoplastics, and post-stroke medications during the IBP. In contrast, NPADM incidence was significantly lower in subjects who were treated with statins, antihypertensives, and non-steroidal bronchodilators during the IBP. While the use of steroids, ${ }^{6}$ benzodiazepines, ${ }^{7}$ anticoagulants, ${ }^{8}$ and non-steroidal anti-inflammatory drugs ${ }^{9}$ are reportedly associated with the development of Alzheimer's disease, our analysis did not detect any significant associations with NPADM.

The results of this study should be interpreted with the understanding that the effects of the target medications on NPADM cannot be separated from those of the underlying conditions. Previous studies have asserted that controlling cardiovascular risk factors can mitigate the onset and progression of Alzheimer's disease. ${ }^{10,11}$ Based on those findings, we included antianginals, antiplatelets, anticoagulants, antihypertensives, and statins in our analysis. However, the multivariate analysis showed that only statins, antihypertensives and non-stroiedal bronchodilators were significantly associated with a lower incidence of NPADM (risk reductions of 18\% for statins and $20 \%$ for antihypertensives). In contrast to our findings, two prospective randomised controlled trials failed to demonstrate the benefits of statins for the prevention of dementia, ${ }^{12,13}$ although beneficial effects were indicated by a cohort study. ${ }^{14}$ Our analysis differed from the two trials in that our subjects were already taking statins during the IBP, whereas statins were prescribed to the trial participants only after randomisation. ${ }^{12,13}$ Next, our analysis found that the use of non-steroidal bronchodilators was associated with a reduced incidence of NPADM. Many researchers suspect that use of medications which have anticholilnergic property is associated with the increased risk of dementia. In the study, the use of non-steroidal bronchodilators which have $\beta$-adrenergic stimulant as well as anti-cholinergic propertiy did not increase NPADM , but reduced it. Meanwile, Japanese package inserts for antidementia medication note that it must be cautiously prescribed to patients who are taking bronchodilators for chronic obstructive pulmonary disease or asthma. ${ }^{15}$ We posit that the reduced incidence of NPADM in subjects being treated with non-steroidal bronchodilators may also be indicative of physicians' caution in prescribing antidementia medications.

Diabetes is a well-established risk factor for dementia, including Alzheimer's disease. ${ }^{1,2}$ Insulin is generally used for patients who are unable to manage their blood glucose levels through the use of oral antidiabetics alone. In addition to its hypoglycaemic properties, oral antidiabetics (sulfonylureas and metformin) have been reported to substantially reduce the risk of dementia. ${ }^{16}$ It is therefore possible that subjects who require insulin have a higher risk of dementia than those taking oral antidiabetics as the latter could mitigate the risk of diabetes-associated dementia. History of stroke is another known risk factor for cognitive decline and its progression to dementia. ${ }^{17}$ In Japan, medications to stimulate brain metabolism or increase cerebrovascular blood flow are prescribed to patients with a history of stroke. Our study subjects who were already taking these post-stroke medications had an elevated risk of NPADM. Next, it is unclear as to whether depression is a risk factor or prodrome of dementia. ${ }^{2}$ Nevertheless, our study subjects who were taking antidepressants during the IBP were found to have a higher incidence of NPADM. Depression is a known risk factor for developing dementia subsequently, Our results, however, do not provide any evidence that antidepressants have any beneficial effects for reversing the depressive symptoms on Alzheimer's disease or Lewy body disease. Finally, the use of antineoplastics were associated with a 
small, but statistically significant, increase in NPADM among our subjects. In contrast, a previous meta-analysis suggested that malignancies can reduce the risk of Alzheimer's disease. ${ }^{18}$ Further research is required to elucidate the effects of antineoplastics on dementia, such as through the induction of injury to neuronal tissue and promotion of the neurodegenerative process. Overall, the results of our study do not contradict the recommendations of the $\mathrm{WHO}^{1}$

Despite being widely prescribed, the currently available anti-dementia medications for Alzheimer's disease and Lewy body disease only provide symptomatic treatment without modifying the underlying neurodegenerative process. ${ }^{2}$ Several phase III trials have failed to demonstrate the effectiveness of candidate drugs in disease modification, and many were terminated before completion. ${ }^{19}$ Trials of aducanumab, an anti-amyloid antibody, were also discontinued after a phase III futility analysis. However, additional data analyses suggest that longer exposure to higher doses may be effective. Although the manufacturer of aducanumab is seeking US Food and Drug Administration approval, it is unclear if the currently available data are sufficient to confirm its effectiveness. ${ }^{20}$ As there are no effective curative treatments at present, the WHO has recommended multidomain strategies that incorporate both lifestyle modifications and pharmaceutical management to reduce new cases of dementia and its progression. ${ }^{1}$ Several prospective randomised trials have assessed the effectiveness of such interventions to prevent cognitive decline, but have produced conflicting results: the FINGER trial reported a reduction in dementia risk, ${ }^{21}$ but two other studies found no significant effects on dementia incidence or cognitive decline. ${ }^{22,23}$

Our study design is characterized by several features. First, under Japan's universal healthcare system, all Kashiwa city residents aged 75 years or older are, without exception, covered by the LLMCS until death, moving out of the city, or admitted to a nursing facility. The LLMCS is managed by a single payer (i.e., the Kashiwa city government). Therefore, a strength of this study is the use of an exhaustive survey of insurance claims data. Second, we used NPADM as the outcome measure because the claims records did not contain any clinical information for other outcomes. A previous study that analysed a different claims dataset from Kashiwa city reported that approximately $19 \%$ of residents aged 75 years or older had a probable diagnosis of dementia, but only $5.8 \%$ were prescribed anti-dementia medications. ${ }^{24}$ It is extremely difficult to obtain accurate diagnoses of Alzheimer's disease or Lewy body disease without access to the complete set of clinical evaluations by physicians, laboratory test results, cognitive test results, and neurological images. It is also essentially impossible to identify the point at which mild cognitive impairment progresses to clinical dementia. For this study, we employed NPADM as a proxy to identify this progression. Third, we focused on older adults (aged $\geq 77$ years) who often have multiple existing conditions that are treated with various medications. We examined 14 categories of medications for commonly observed conditions in the target population. In order to ensure the accuracy of identifying patients with specific conditions in the target population, we only used prescription data that are directly linked with reimbursements. Diagnostic codes were not used to identify patients with specific conditions due to the possibility that providers may assign these codes for the sole purpose of reimbursement. We believe this approach effectively utilizes the accurate and reliable information available in Japanese claims records. Efforts to integrate claims records and clinical information from electronic health records are needed to support more accurate population studies that would shed light on the effectiveness and safety profile of various therapies. The Pharmaceuticals and Medical Devices Agency of Japan established the Medical Information Database Network (MID-NET) Project to integrate these datasets in selected medical institutions, and is being used for post-marketing surveillance of marketing authorization holder products. ${ }^{25}$ 
An important consideration of our findings is their generalizability to the overall Japanese population or other countries. Environmental factors (such as social network size) in urban areas may be different from those in more rural regions. For example, the Hisayama Study is a population-based cohort study of a rural Japanese town (mean age: 76 years ) that examines the risk factors for various diseases, including dementia. ${ }^{26}$ The reported prevalence of Alzheimer's disease in the Hisayama Study (12.3\%) is higher than that of our study, which may be explained by the differences in primary endpoint. Alzheimer's disease was diagnosed in the Hisayama Study through a combination of clinical assessments, imaging tests, and cognitive tests such as the Mini-Mental State Examination in accordance with the Diagnostic and Statistical Manual of Mental Disorders, Third Edition. In contrast, our study relied on the initiation of anti-dementia medications to identify disease onset. With respect to the generalizability of our findings to other countries, considerations must be made toward the differences in healthcare delivery systems, social systems, education systems, and ethnic factors. ${ }^{27}$ Several higher-income countries (including the US, the UK, Sweden, the Netherlands, and Canada) have reported recent reductions in dementia incidence, ${ }^{2}$ but the prevalence of dementia is increasing in Japan. ${ }^{28}$ Although our findings are consistent with WHO recommendations, their extrapolation to other countries should be carefully considered due to these inherent differences.

\section{Limitations}

The study has several limitations. First, administrative claims collected in real world clinical practice are not designed for research purpose. Therefore, medical information including accurate diagnostic criteria for dementia was not included. Although the information on the use of prescription medications during the IBP was accurate, the exposure durations before the IBP were unknown. As we could not ascertain the delivered dose of each drug, our analysis did not take the dose-response effect into account. Second, the effects of each medication could not be distinguished from the underlying pathophysiological condition for which it was prescribed. For example, NPADM in patients taking antidiabetics may be affected by the medication, diabetes, or a combination thereof. In addition, the control of blood glucose levels is likely to be an important aspect of reducing dementia risk, but this information was not available in our dataset. Third, although we assume that NPADM captured the progression from asymptomatic into symptomatic dementia, it is entirely dependent upon the decisions of individual physicians, such as neurologists, psychiatrists, and primary care physicians. Various guidelines include recommendations on the prescription and discontinuation of cholinesterase inhibitors and N-methyl-d-aspartate receptor antagonists. ${ }^{29,30}$ However, there may be variations in the decision-making process among subspecialties and physicians. Fourth,there is a discussion that anticholinergic medications are associated with risk of dementia $^{31}$. It is not yet well undrstood that what level of anticholinergic property and what class of medication have increased risk of dementia. We included antidepressant and bronchodilator which have anticholinergic properties, while other class of drugs such as for parkinson disease, and for peptic ulcer were not included in the study. To estimate the level of anticholinergic in each drug and to evaluated the effect on NPADM are out of scope in the present study. . Fifth, although anti-dementia medications are only approved for Alzheimer's disease and Lewy body disease in Japanese regulatory approval, we cannot exclude the possibility that physicians may prescribe these medications to treat vascular dementia. As the study population is very old adults (>76 year old), it is supposed that substantial proportion of study subjects have combined pathology of vascular dementia as well as Alzheimer disease and Lewy body disease. Although, post-stroke medication prescribed for history of stroke reflected the vascular component of dementia, no obvious history of stroke such as multiple lacunar infarcts may be overlooked in the study. Finally, $2.1 \%$ of study subjects $(n=879)$ were censored because they were admitted into nursing facility during study period because they were covered by the long-term care insurance system for disabled 
subjects after admission. Most of them were admitted into nursing home due to the impairment of physical function. Some of them might have concomitant cognitive decline. As incidence of NPADM in older adults who reside in nursing facility is assumed to be higher in comparison with community-dwelling subjects, the effect of medications assessed in the study might have been underestimated.

\section{Conclusions}

In this complete enumeration study of insurance claims records from older adults, we examined the associations between the use of prescription medications for existing chronic diseases and NPADM. The findings of this population-based natural experiment were generally consistent with WHO recommendations for the control of dementia risk factors. The appropriate use of statins and effective management of hypertension appeared to reduce NPADM. Our study may provide a benchmark for the mitigation of the risk of progression from pre-clinical dementia to symptomatic Alzheimer's disease or Lewy body disease in older adults. Although our study's scope did not include the value of dementia risk mitigation or cost-effectiveness assessments, such analyses may be feasible using the approach described here. We believe that our findings and similar studies have potential applications in evidence-based healthcare policymaking.

(Clinical Trial Registration: UMIN-CTR UMIN000039040)

\section{Abbreviations}

\begin{tabular}{|l|l|}
\hline NPADM & New prescription of anti-dementia medication \\
\hline LLMCS & Long Life Medical Care System in Japan \\
\hline IBP & "initial background period" of three months from April 1 to June 30, 2012 \\
\hline WHO & World Health Organization \\
\hline
\end{tabular}

\section{Declarations}

\section{Ethics approval and consent to participate}

The present research involving human data were performed in accordance with the Declration of Helsinki. The study used anonymized insurance claims records without opt-in informed consent, and was approved by the Kashiwa city government. The study was also approved by the institutional ethics committees of Tokyo Metropolitan Institute of Gerontology (Approval No28: October 16, 2015) where this study was performed.

\section{Consent for publication}

As there are no details on individuals reported within the manuscript, authors think that consent for publication is not required.

\section{Availability of data material (Data sharing)}

All data underlying this study are owned by the Kashiwa city government. The study was conducted in accordance with a contract between the Kashiwa city government and the Tokyo Metropolitan Geriatric Medical Center and Institute of Gerontology. The contract stipulates that the authors are prohibited from using the data for any purpose other than this study or providing the data to anyone other than the approved research group members without the express permission of the Kashiwa city government. Researchers interested in the data should contact the project leader, Dr. Ishizaki. 


\section{Competing interests}

Nobuhiro Handa has nothing to disclose.

Seigo Mitsutake has nothing to disclose.

Tatsuro Ishizaki has nothing to disclose.

Tetsuo Nakabayashi has nothing to disclose.

Masahiro Akishita reports grants from Astellas Pharma, grants from Bayer HealthCare, grants from Boehringer Ingelheim, grants from Chugai Pharmaceutical, grants and personal fees from Daiichi Sankyo, grants from Eli Lilly Japan, grants from Eisai, grants from Kracie Pharma, grants and personal fees from Mitsubishi-Tanabe Pharma, grants and personal fees from MSD, grants from Novartis Japan, grants from Ono Pharmaceutical, grants from Sanofi, grants and personal fees from Takeda Pharmaceutical, grants from Teijin Pharma, grants from Tsumura, and personal fees from Sumitomo Dainippon Pharma, outside the submitted work.

Nanako Tamiya has nothing to disclose.

Satoru Yoshie has nothing to disclose.

Katsuya lijima has nothing to disclose.

\section{Funding}

This research did not receive any specific grant from funding agencies in the public, commercial, or not-for-profit sectors. The cost of conducting the study was covered by the general budget of Tokyo Metropolitan Institute of Gerontology. There is no particular funding number available

\section{Author contribution:}

Nobuhiro Handa:

the conception and design of the work, the data acquisition, analysis, interpretation of data, medical writing, editing the manuscript

Seigo Mitsutake:

the conception and design of the work, the data acquisition, analysis, interpretation of data, critical revision, editing the manuscript

Tatsuro Ishizaki:

the conception and design of the work, the acquisition, analysis, or interpretation of data for the work, editing the manuscript, critical revision, final approveal

Tetsuo Nakabayashi

Analysis, or interpretation of data for the work, editing the manuscript, critical revision 
Masahiro Akishita

Analysis, or interpretation of data for the work, editing the manuscript, critical revision

Nanako Tamiya

Analysis, or interpretation of data for the work, editing the manuscript, critical revision

Satoru Yoshie

Analysis, or interpretation of data for the work, editing the manuscript, critical revision

Katsuya lijima

Analysis, or interpretation of data for the work, editing the manuscript, critical revision

\section{Acknowledgments}

We thank Dr. Syunei Kyo for his support to our research team. The views expressed in this article are those of the authors, and do not necessarily reflect the official views of the PMDA.

\section{References}

1. Risk Reduction of Cognitive Decline and Dementia: WHO Guidelines https://apps.who.int/iris/bitstream/handle/10665/312180/9789241550543-eng.pdf?ua=1 World health Organization; 2019 [accessed 15 July 2020]

2. Livingston G, Sommerlad A, Orgeta V, Costafreda SG, Huntley J, Ames D et al. Dementia, prevention, intervention, and care. Lancet. 2017; 390: 2673-2734. doi: 10.1016/S0140-6736(17)31363-6.

3. Joe E, Ringman JM. Cognitive symptoms of Alzheimer's disease: clinical management and prevention. BMJ. 2019;367:I6217. doi: 10.1136/bmj.I6217.

4. Outline of Health Care Insurance System. https://www.mhlw.go.jp/english/wp/wp-hw3/dl/2-001.pdf April 20090; [accessed 15 July 2020]

5. Mitsutake S, Ishizaki T, Teramoto C, Shimizu S, Ito H. Patterns of Co-Occurrence of Chronic Disease Among Older Adults in Tokyo, Japan. Prev Chronic 2019; 16: E11. doi: 10.5888/pcd16.180170

6. Nerius M, Haenisch B, Gomm W, Doblhammer G, Schneider A. Glucocorticoid therapy is associated with a lower risk of dementia. J Alzheimers Dis. 2020;73(1):175-183. doi: 10.3233/JAD-190444.

7. Ding M, Fratiglioni L, Johnell K, Santoni G, Fastbom J, Ljungman P, et al. Atrial fibrillation, antithrombotic treatment, and cognitive aging: A population-based study. 2018; 91: e1732-e1740. doi: 10.1212/WNL.0000000000006456

8. Zhong G, Wang Y, Zhang Y, Zhao Y. Association between Benzodiazepine Use and Dementia: A Meta-Analysis. PLoS One 2015; 10: e0127836. doi: 10.1371/journal.pone.0127836. eCollection 2015

9. Wang J, Tan L, Wang HF, Tan CC, Meng XF, Wang C, et al. Anti-inflammatory drugs and risk of Alzheimer's disease: an updated systematic review and meta-analysis. J Alzheimers Dis 2015; 44: 385-96. doi: 10.3233/JAD-141506. 
10. Samieri C, Perier MC, Gaye B, Proust-Lima C, Helmer C, Dartigues JF et al. Association of Cardiovascular Health Level in Older Age With Cognitive Decline and Incident Dementia. 2018; 320: 657-664. doi: 10.1001/jama.2018.11499.

11. Sabia S, Fayosse A, Dumurgier J, Schnitzler A, Empana JP, Ebmeier KP, et al. Association of ideal cardiovascular health at age 50 with incidence of dementia: 25 year follow-up of Whitehall II cohort study. BMJ. 2019;366:I4414. doi: 10.1136/bmj.I4414.

12. Heart Protection Study Collaborative Group. MRC/BHF Heart Protection Study of cholesterol lowering with simvastatin in 20536 high-risk individuals: a randomised placebo-controlled trial.Lancet 2002; 360: 7-22. doi: 10.1016/S0140-6736(02)09327-3.

13. Shepherd J, Blauw GJ, Murphy MB, Bollen EL, Buckley BM, Cobbe SM, et al. Pravastatin in elderly individuals at risk of vascular disease (PROSPER): a randomised controlled trial. Lancet 2002; 360: 1623-30. doi: 10.1016/s0140-6736(02)11600-x.

14. Zhang X, Wen J, Zhang Z. Statins use and risk of dementia: A dose-response meta analysis. Medicine (Baltimore). 2018;97:e11304. doi: 10.1097/MD.0000000000011304.

15. Package inserts: Aricept (Japanese) https://www.info.pmda.go.jp/go/pack/1190012C1020_1_27/ May 2017; [accessed 15 July 2020]

16. Hsu CC, Wahlqvist ML, Lee MS, Tsai HN. Incidence of Dementia is Increased in Type 2 Diabetes and Reduced by the Use of Sulfonylureas and Metformin. J Alzheimers Dis. 2011;24:485-93. doi: 10.3233/JAD-2011101524.

17. Zhou J, Yu JT, Wang HF, Meng XF, Tan CC, Wang J, et al. Association between stroke and Alzheimer's disease: systematic review and meta-analysis. J Alzheimers Dis. 2015;43(2):479-89. doi: 10.3233/JAD-140666.

18. Zhang Q, Guo S, Zhang X, Tang S, Shao W, Han X et al. Inverse relationship between cancer and Alzheimer's disease: a systemic review meta-analysis. Neurol Sci 2015;36:1987-94.doi: 10.1007/s10072-015-2282-2.

19. Cummings J, Lee G, Ritter A, Sabbagh M, Zhong K. Alzheimer's disease drug development pipeline: 2019. Alzheimers Dement (N Y). 2019;5:272-293. doi: 10.1016/j.trci.2019.05.008. eCollection 2019.

20. Howard R, Liu KY. Questions EMERGE as Biogen claims aducanumab turnaround. Nat Rev Neurol. 2020;16:6364. doi: 10.1038/s41582-019-0295-9.

21. Ngandu T, Lehtisalo J, Solomon A, Levälahti E, Ahtiluoto S, Antikainen R et al. A 2 year multidomain intervention of diet, exercise, cognitive training, and vascular risk monitoring versus control to prevent cognitive decline in at-risk elderly people (FINGER): a randomised controlled trial Lancet. 2015; 385: $2255-63$. doi: 10.1016/S0140-6736(15)60461-5.

22. Moll van Charante EP, Richard E, Eurelings LS, van Dalen JW, Ligthart SA, van Bussel EF, et al. Effectiveness of a 6-year multidomain vascular care intervention to prevent dementia (preDIVA): a cluster-randomised controlled trial. Lancet. 2016; 388: 797-805. doi: 10.1016/S0140-6736(16)30950-3.

23. Andrieu S, Guyonnet S, Coley N, Cantet C, Bonnefoy M, Bordes S et al. Effect of long-term omega 3 polyunsaturated fatty acid supplementation with or without multidomain intervention on cognitive function in elderly adults with memory complaints (MAPT): a randomized, placebo-controlled trial. Lancet Neurol. 2017; 16:377-389. doi: 10.1016/S1474-4422(17)30040-6

24. Kuroda N, Hamada S, Sakata N, Jeon B, lijima K, Yoshie S, et al. Antipsychotic use and related factors among people with dementia aged 75 years or older in Japan: A comprehensive population-based estimation using medical and long-term care data. Int J Geriatr Psychiatry. 2019;34:472-479. doi: 10.1002/gps.5041.

Page $13 / 20$ 
25. Yamaguchi M, Inomata S, Harada S, Matsuzaki Y, Kawaguchi M, Ujibe M, et al. Establishment of the MID-NET ${ }_{\circledast}$ medical information database network as a reliable and valuable database for drug safety assessments in Japan. Pharmacoepidemiol Drug Saf. 2019;28:1395-1404. doi: 10.1002/pds.4879.

26. Ninomiya T. Japanese Legacy Cohort Studies: The Hisayama Study. J Epidemiol. 2018;28:444-451. doi: 10.2188/jea.JE20180150.

27. Zissimopoulos JM, Barthold D, Brinton RD, Joyce G. Sex and Race Differences in the Association Between Statin Use and the Incidence of Alzheimer Disease. JAMA Neurol. 2017; 74: 225-232. doi:10.1001/jamaneurol.2016.3783.

28. Okamura H, Ishii S, Ishii T, Eboshida A. Prevalence of dementia in Japan: a systematic review. Dement Geriatr Cogn Disord 13;36:111-8. doi: 10.1159/000353444.

29. Dementia: assessment, management and support for people living with dementia and their carers. NICE guideline. https://www.nice.org.uk/guidance/ng97 20 June 2018 [accessed 15 July 2020]

30. Practice guideline update summary: Mild cognitive impairment. Report of the Guideline Development, Dissemination, and Implementation Subcommittee of the American Academy of Neurology Neurology 2018;90:126-135. doi:10.1212/WNL.0000000000004826

31. Richardson K, Fox C, Maidment I. Anticholinergic drugs and risk of dementia: case-control study. BMJ 2018;361:k1315 | doi: 10.1136/bmj.k1315. doi: 1136/bmj.k1315

\section{Figures}




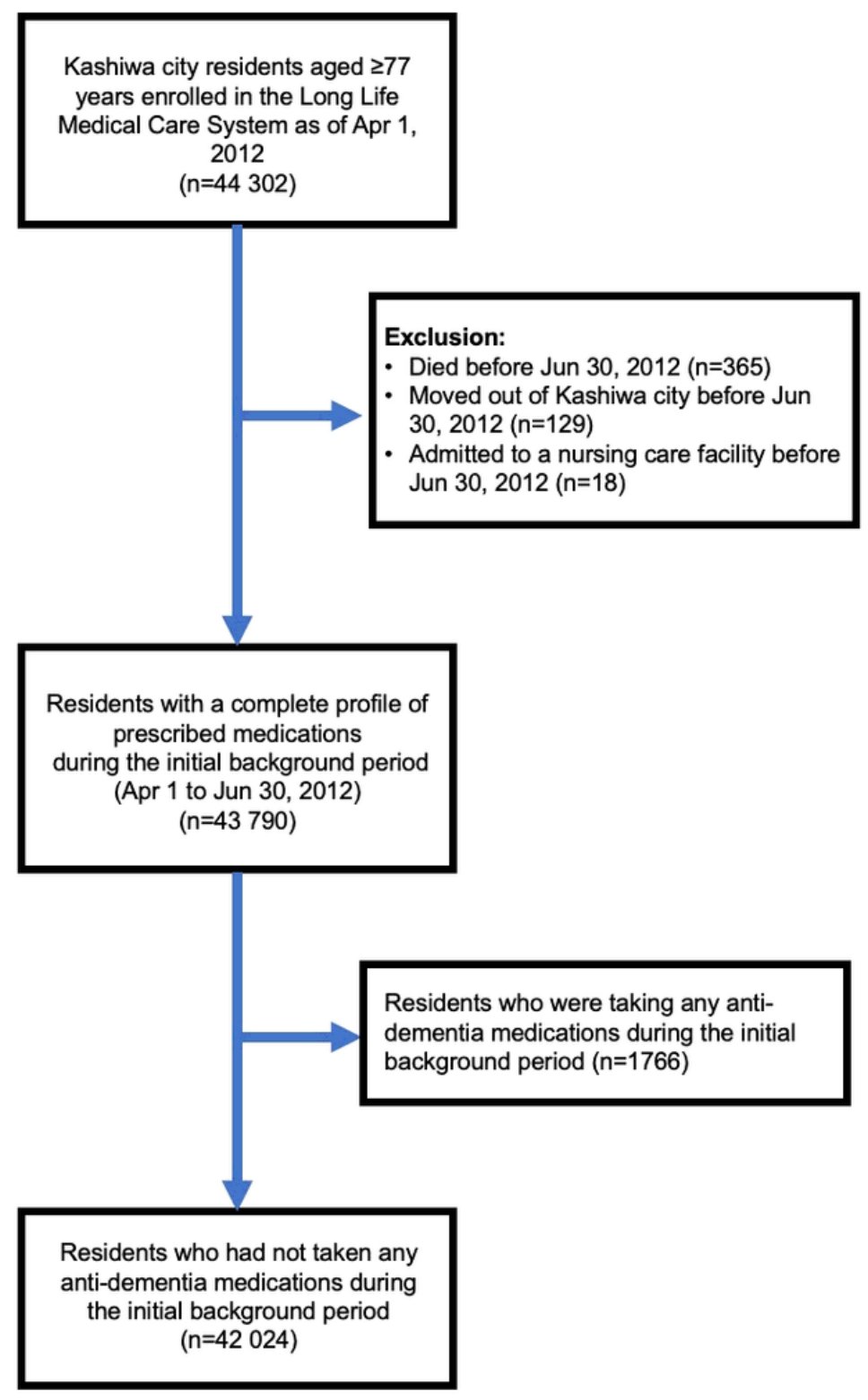

Figure 1

Study flow diagram Initial background period: From April 1st 2012 to Jun 31st 2012 
Figure 2A

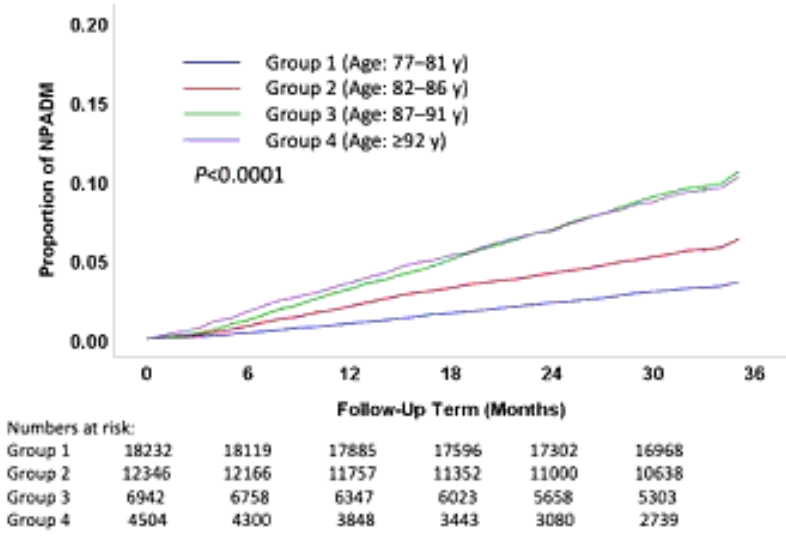

Figure $2 \mathrm{C}$

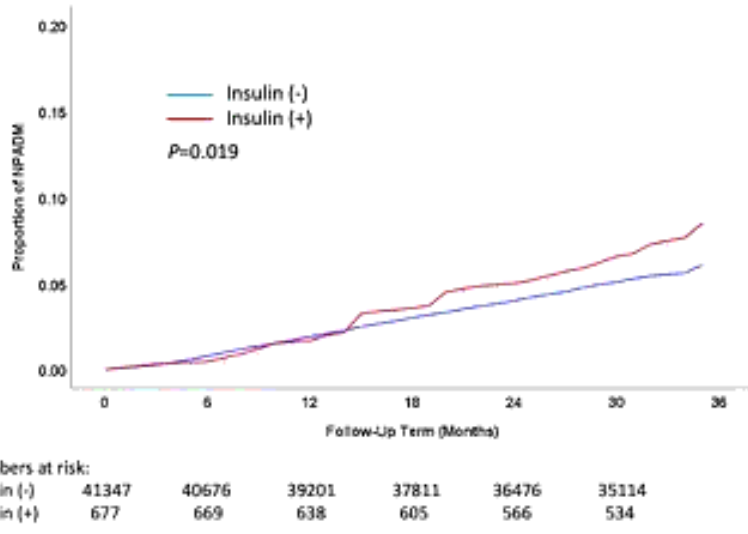

Figure 2B

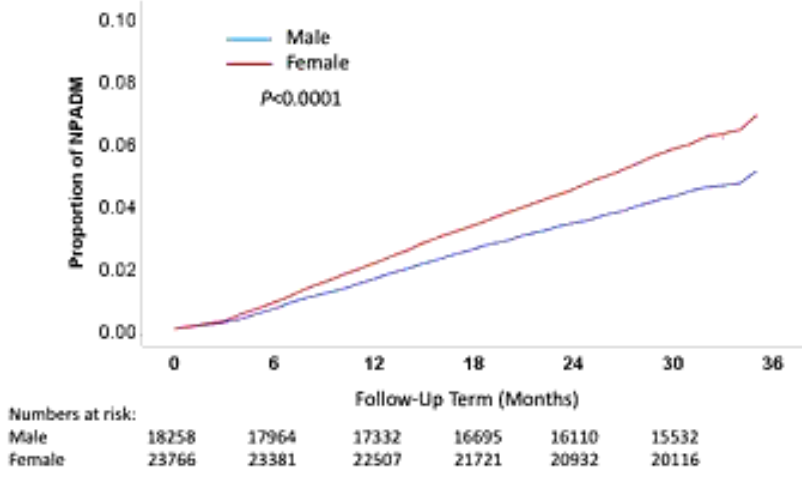

Figure 2D

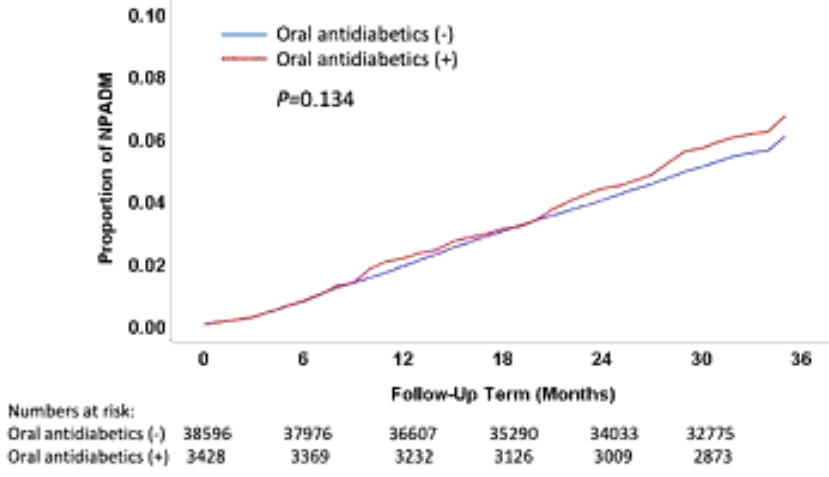

Figure 2

Kaplan-Meier curves showing the cumulative incidence of NPADM stratified by subject characteristics and the use of antidiabetics Cumulative incidence of NPADM stratified by (A) age groups, (B) sex, (C) use of insulin, and (D) use of oral antidiabetics during the IBP. IBP=initial background period (April 1 to June 30, 2012), NPADM=New prescription of anti-dementia medication. 
Figure 3A

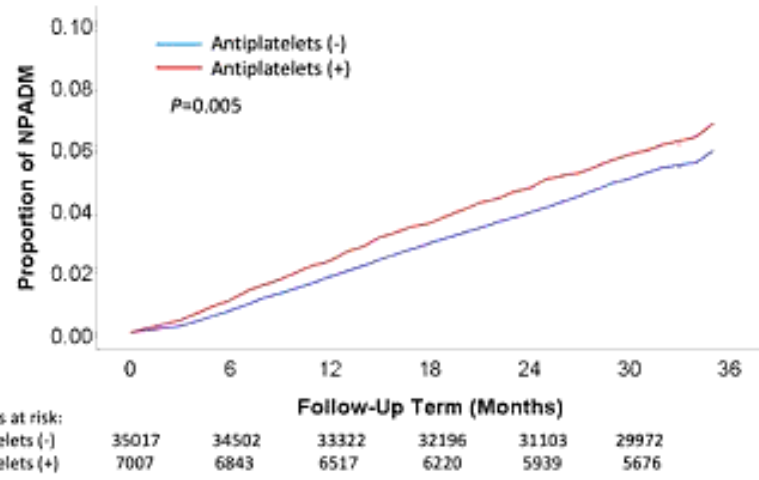

Figure $3 C$

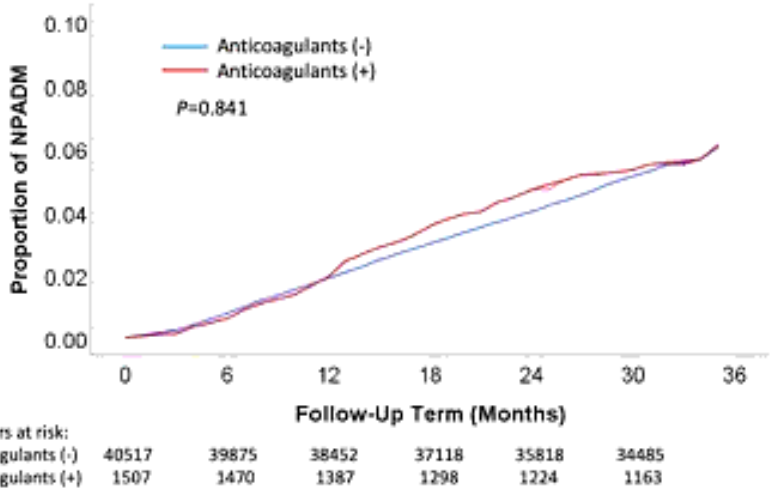

Figure 3B

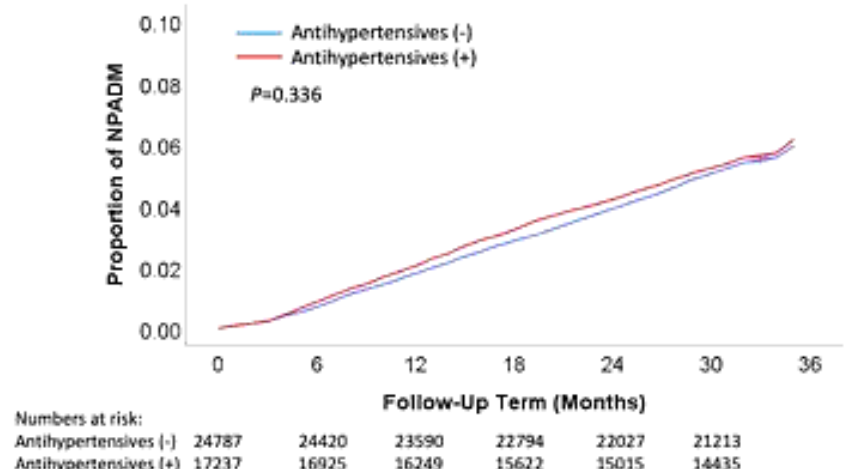

Figure 3D

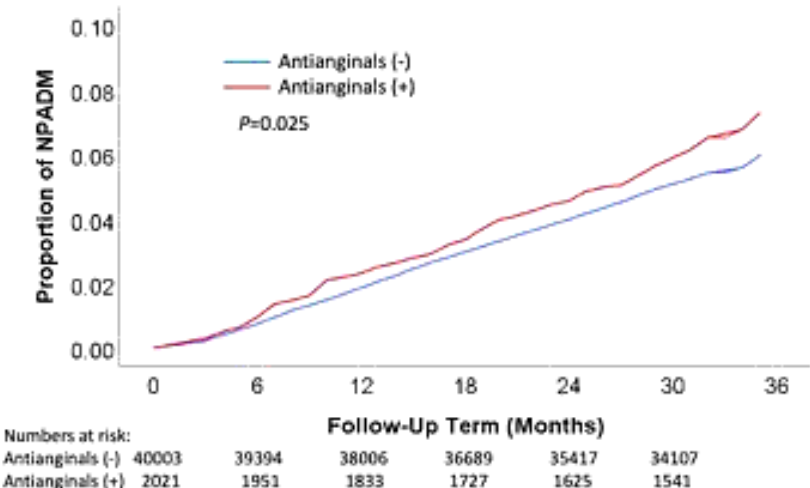

Figure 3

Kaplan-Meier curves showing the cumulative incidence of NPADM stratified by the use of blood and cardiovascular system medications Cumulative incidence of NPADM stratified by the use of (A) antiplatelets, (B) antihypertensives, (C) anticoagulants, and (D) antianginals during the IBP. IBP=initial background period (April 1 to June 30, 2012), NPADM=New prescription of anti-dementia medication. 
Figure 4A

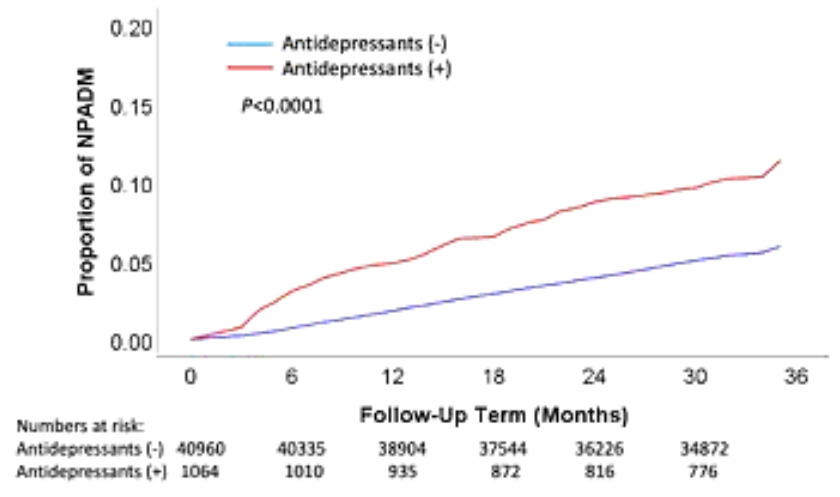

Figure $4 \mathrm{C}$

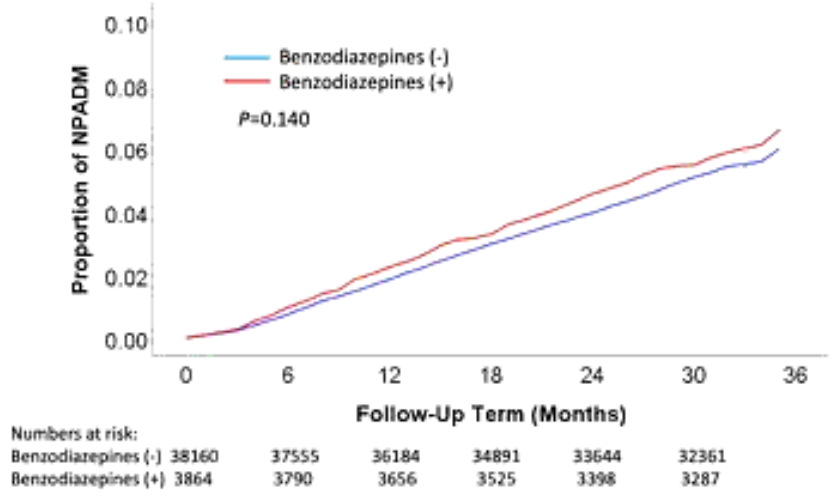

Figure 4B

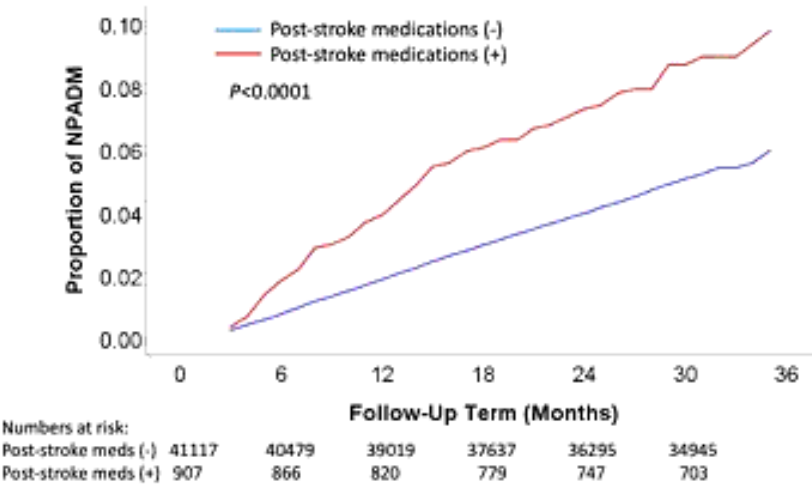

Figure 4D

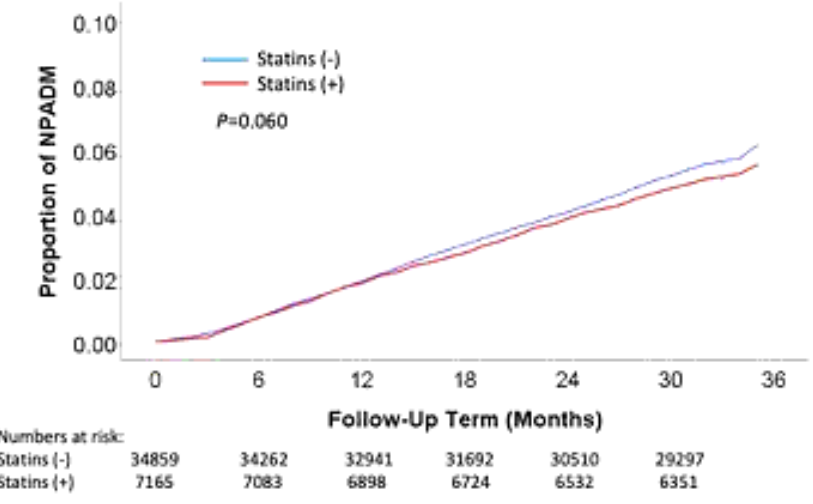

\section{Figure 4}

Kaplan-Meier curves showing the cumulative incidence of NPADM stratified by the use of brain/nervous system and dyslipidaemia medications Cumulative incidence of NPADM stratified by the use of (A) antidepressants, (B) post-stroke medications, (C) benzodiazepines, and (D) statins during the IBP IBP=initial background period (April 1 to June 30, 2012), NPADM=New prescription of anti-dementia medication. 
Figure 5A

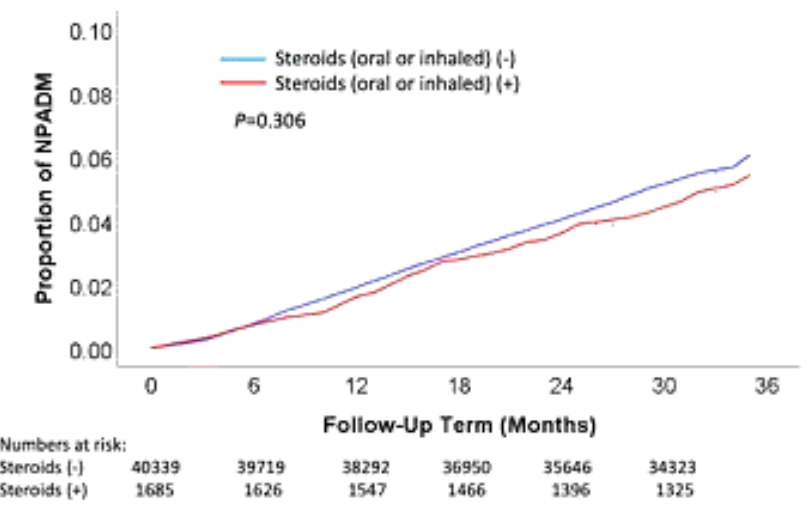

Figure 5C

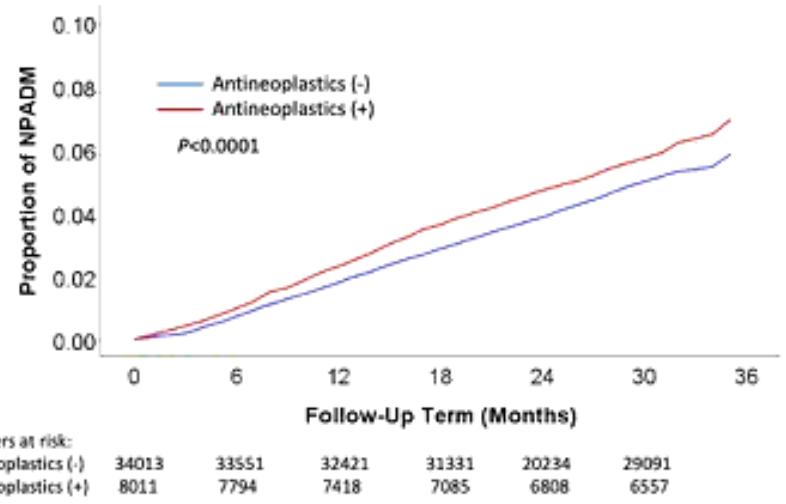

Figure 5B

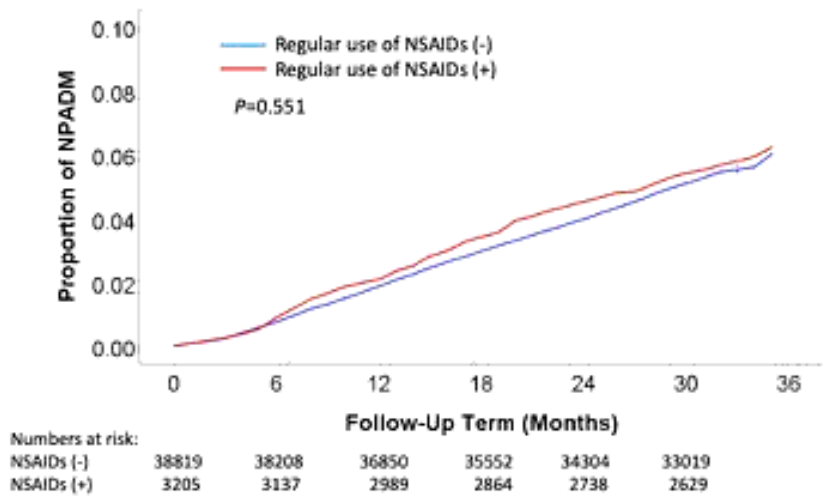

Figure 5D

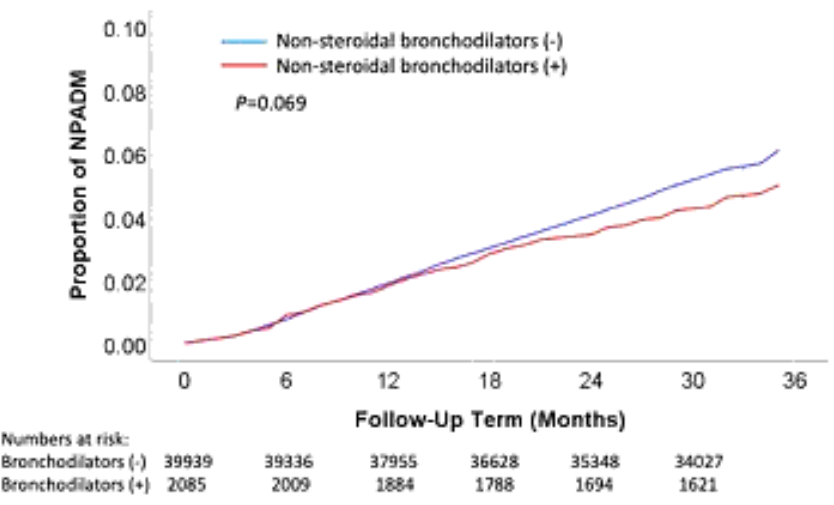

\section{Figure 5}

Kaplan-Meier curves showing the cumulative incidence of NPADM stratified by the use of steroids, antiinflammatory drugs, antineoplastics, and bronchodilators Cumulative incidence of NPADM stratified by the use of (A) steroids (oral or inhaled), (B) NSAIDs, (C) antineoplastics, and (D) non-steroidal bronchodilators during the IBP IBP=initial background period (April 1 to June 30, 2012), NPADM=New prescription of anti-dementia medication, NSAID=non-steroidal anti-inflammatory drug. 


$\begin{array}{lccc}\text { Age: } 77-81 \text { years } & 18232 & \text { Reference } & \text { Reference } \\ 82-86 \text { years } & 12346 & 1.90 & {[1.70-2.12]} \\ 87-91 \text { years } & 6942 & 3.22 & {[2.87-3.61]} \\ \geq 92 \text { years } & 4504 & 3.05 & {[2.67-3.49]} \\ \text { Female } & 23766 & 1.26 & {[1.15-1.37]} \\ \text { Antidepressants } & 1064 & 1.79 & {[1.47-2.18]} \\ \text { Post-stroke medications } & 907 & 1.45 & {[1.16-1.82]} \\ \text { Insulin } & 677 & 1.34 & {[1.01-1.78]} \\ \text { Antineoplastics } & 8011 & 1.12 & {[1.01-1.24]} \\ \text { Oral antidiabetics } & 3428 & 1.11 & {[0.95-1.29]} \\ \text { Antiplatelets } & 7007 & 1.09 & {[0.96-1.23]} \\ \text { Antianginals } & 2021 & 1.04 & {[0.86-1.25]} \\ \text { Anticoagulants } & 1507 & 0.95 & {[0.76-1.19]} \\ \text { Benzodiazepines } & 3864 & 0.91 & {[0.79-1.05]} \\ \text { NSAIDs, regular use } & 3205 & 0.87 & {[0.74-1.03]} \\ \text { Statins } & 7165 & 0.82 & {[0.73-0.92]} \\ \text { Steroids, oral or inhaled } & 1685 & 0.81 & {[0.65-1.01]} \\ \text { Antihypertensives } & 17237 & 0.80 & {[0.73-0.88]} \\ \text { Non-steroidal bronchodilators } & 2085 & 0.72 & {[0.58-0.88]}\end{array}$

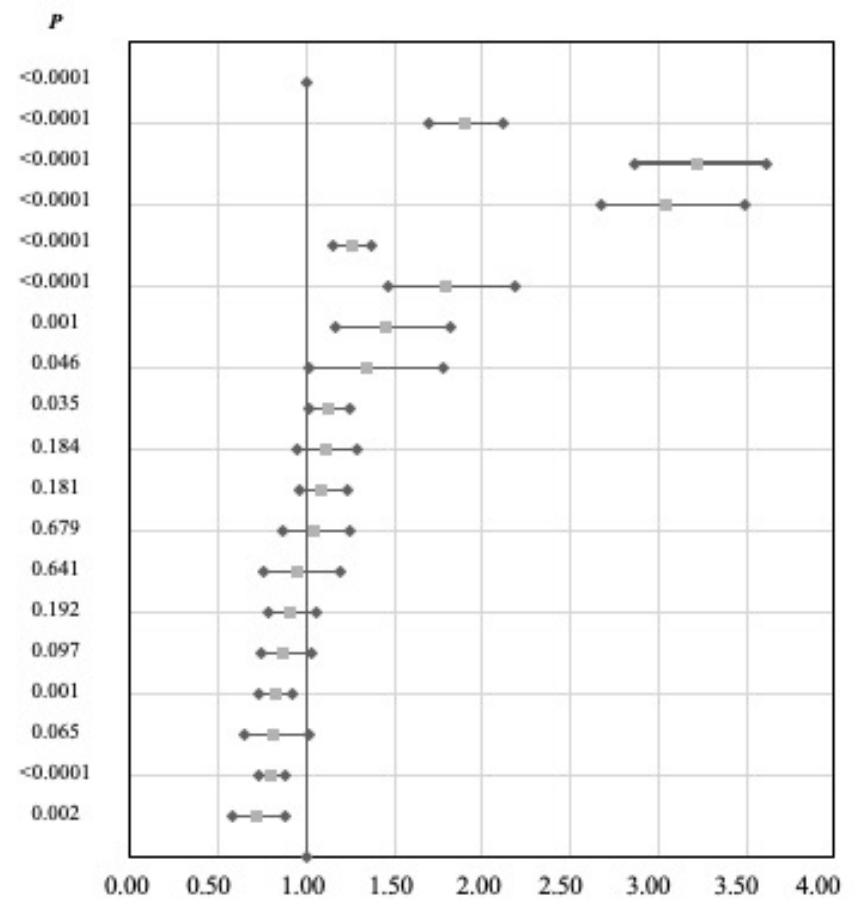

Figure 6

Results of the Cox proportional hazards analysis of NPADM incidence $\mathrm{Cl}=$ confidence interval, NSAID=non-steroidal anti-inflammatory drug.

\section{Supplementary Files}

This is a list of supplementary files associated with this preprint. Click to download.

- Supplementalfile12.pdf 